\title{
Wavelength dependence of efficiency limiting mechanisms in Type-I Mid-infrared $\mathrm{GaInAsSb} / \mathrm{GaSb}$ lasers
}

\author{
Timothy D. Eales, Igor P. Marko, Barnabas A. Ikyo, Alf R. Adams, Life Fellow, IEEE, Shamsul \\ Arafin, Member, IEEE, Stephan Sprengel, Member, IEEE, Markus-C. Amann, Fellow, IEEE, and \\ Stephen J. Sweeney, Senior Member, IEEE
}

\begin{abstract}
The efficiency limiting mechanisms in type-I GaInAsSb-based quantum well (QW) lasers, emitting at $2.3 \mu \mathrm{m}$, 2.6 $\mu \mathrm{m}$ and $2.9 \mu \mathrm{m}$, are investigated. Temperature characterization techniques and measurements under hydrostatic pressure identify an Auger process as the dominant non-radiative recombination mechanism in these devices. The results are supplemented with hydrostatic pressure measurements from three additional type-I GaInAsSb lasers, extending the wavelength range under investigation from 1.85-2.90 $\mu \mathrm{m}$. Under hydrostatic pressure, contributions from the CHCC and CHSH Auger mechanisms to the threshold current density can be investigated separately. A simple model is used to fit the non-radiative component of the threshold current density, identifying the dominance of the different Auger losses across the wavelength range of operation. The CHCC mechanism is shown to be the dominant non-radiative process at longer wavelengths $(>2 \mu \mathrm{m})$. At shorter wavelengths $(<2 \mu \mathrm{m})$ the $\mathrm{CHSH}$ mechanism begins to dominate the threshold current, as the bandgap approaches resonance with the spin-orbit split-off band.
\end{abstract}

Index Terms-Auger recombination, Interband Mid-IR lasers, MIR devices, Quantum well lasers.

\section{INTRODUCTION}

$\mathrm{T}$ HE pursuit of semiconductor lasers operating in the midinfrared is motivated by a variety of applications, which include environmental monitoring, non-invasive medical diagnosis and free-space communications [1]-[3]. Developments in material quality and structural design over several decades have established the type-I active region as the most competitive laser geometry in the $2-3 \mu \mathrm{m}$ spectral range [4]. High performance lasers based on the GaInAsSb/GaSb material system have reported Watt-level output powers up to

Details of the data and how to request access are available from the University of Surrey publications repository at http://epubs.surrey.ac.uk/. This work was supported in part by the EPSRC (UK) projects EP/H005587/01, EP/H050787/1 and EP/N021037/1, Benue State University Nigeria and the Petroleum Technology Development Fund Nigeria, for sponsorship of the PhD Studentship of B. A. Ikyo. T. D. Eales acknowledges EPSRC (UK) for sponsorship of a PhD studentship.

T. D. Eales, I. P. Marko, A. R. Adams and S. J. Sweeney are with the Advanced Technology Institute, University of Surrey, Guildford, Surrey, GU2 7XH, United Kingdom. (email: t.eales@surrey.ac.uk; i.marko@surrey.ac.uk; a.adams@surrey.ac.uk; s.sweeney@surrey.ac.uk). B. A. Ikyo was with the
$2.5 \mu \mathrm{m}[5]$ and hundreds of milli-Watts at wavelengths past $3 \mu \mathrm{m}[6]$. Currently, the longest wavelengths accessible to typeI GaInAsSb lasers operating at room temperature are $3.44 \mu \mathrm{m}$ in continuous wave $(\mathrm{CW})$ mode [7] and $3.73 \mu \mathrm{m}$ in pulsed mode [8]. However, the threshold current density of type-I devices remains subject to a high degree of temperature sensitivity. This is most significant at longer wavelengths, where thermal stability has been found to deteriorate rapidly with increasing wavelength [9].

The efficiency limiting mechanisms in type-I devices are typically identified as Auger recombination, optical losses such as inter-valence band absorption (IVBA) and carrier leakage [10]. In an Auger process the energy released from electronhole recombination is transferred to a third carrier which thermally de-excites, depositing energy into the lattice. Between 2-3 $\mu \mathrm{m}$ the direct $\mathrm{CHCC}$ and CHSH Auger processes can both exert an influence. In the CHCC process, a Conduction band electron recombines with a Heavy hole, exciting a second Conduction band electron into a higher energy Conduction band state. The probability of the CHCC process is expected to increase approximately exponentially with increasing wavelength and is therefore extremely detrimental to device performance in narrow bandgap devices [11]. In the CHSH process the recombination of a Conduction band electron and a Heavy hole excites an electron in the Spin-orbit split-off band to the Heavy hole band. The CHSH process has a dramatically enhanced probability at wavelengths where the bandgap, $E_{g}$, approaches resonance with the spin-orbit split-off energy, $\Delta_{S O}$ [12]. An increase in the CHSH process is often accompanied by an increase in inter-valence band absorption where an electron in the spin-orbit split-off band is promoted to a state in the heavy hole band through the absorption of a photon [13]. A third Auger process that may be considered is the CHLH Auger

Advanced Technology Institute, University of Surrey, Guildford, Surrey, GU2 7XH, United Kingdom. He is now with Benue State University, Makurdi, PMB 102119 Makurdi, Nigeria. (email: aikyo@ bsum.edu.ng).

S. Arafin was with the Walter Schottky Institut, Technische Universität München, Am Coulombwall 3, 85748 Garching, Germany. He is now with the Department of Electrical and Computer Engineering, University of California at Santa Barbara, Santa Barbara, CA 93106, USA. (email: sarafin@ece.ucsb.edu).

S. Sprengel and M. C. Amann are with the Walter Schottky Institut, Technische Universität München, Am Coulombwall 3, 85748 Garching, Germany. (email: stephan.sprengel@wsi.tum.de; amann@wsi.tum.de). 
process. However, in compressively strained quantum well devices such as those considered here, the CHLH process is effectively suppressed since energy and momentum conservation cannot be satisfied. The CHCC Auger process therefore dominates over the CHLH process in the 2-3 $\mu \mathrm{m}$ wavelength range [11], [14]. Carrier leakage has also been identified as a major contributor to the temperature instability of the threshold current in GaInAsSb devices, with some reports of it exceeding Auger recombination as the dominant nonradiative channel [15], [16]. In mid-infrared GaInAsSb devices, carrier leakage occurs mainly through the valence band due to the small valence band offset between the quantum well and barrier layers. Improved carrier confinement has been achieved independently, with both increased levels of compressive strain, and through the larger valence band offset offered by quinternary AlGaInAsSb barriers [17], [18]. Improvements in carrier confinement has been the main pathway in achieving room temperature and $\mathrm{CW}$ operation well above $3 \mu \mathrm{m}$. Any subsequent reduction in the threshold carrier density, $n_{t h}$, also has a considerable effect on Auger recombination rates, due to its carrier density cubed dependence. Consequently, advances in device structure and material quality are important pathways for continued progresses in type-I quantum well devices [19], [20].

An alternative approach for lasers operating in the midinfrared is inter-subband quantum cascade lasers (QCLs). In these devices, photons are generated by electron transitions between confined states in the conduction band, effectively eliminating Auger recombination and offering a high degree of tunability within the same material system [21]. These properties, combined with the enhanced carrier efficiency provided by the cascading scheme have allowed QCLs to achieve lasing across the mid-infrared spectral region, with high performance between 4-12 $\mu \mathrm{m}$ [22]. There are, however, significant challenges for shorter wavelength QCLs, as high conduction band offsets (> $400 \mathrm{meV}$ ) are necessary to confine the electron states [23]. Several material systems have been proposed with notable successes including lasing at $2.67 \mu \mathrm{m}$ in InAs/AlSb based QCLs on InAs, and continuous wave, room temperature operation at $3 \mu \mathrm{m}$ in GaInAs/AlInAs QCLs on InP [24], [25]. The performance of these devices is fundamentally limited by inter-valley scattering in the conduction band. These effects limit the maximum operating temperature and further reduce temperature stability [26]. Inter-valley scattering becomes more pronounced for shorter wavelength devices and has obstructed the development of high performance, room temperature QCLs between 2-3 $\mu \mathrm{m}$.

A third approach for mid-infrared lasers is type-II inter-band lasers. Currently high performance type-II lasers occupy the spectral niche between type-I lasers and QCLs. However, the wavelengths accessible by type-II lasers has expanded, recently achieving operation $<3 \mu \mathrm{m}$ [27]. As in conventional diode lasers, photon emission is achieved through the radiative recombination of electrons and holes. Type-II lasers are typically integrated within a cascading scheme which benefit from the improved efficiency of carrier recycling at multiple stages. The type-II geometry confines electrons and holes into spatially separated regions, reducing the electron-hole overlap and consequently the Auger recombination rate [28]. However, this effect is accompanied by a reduction in radiative recombination compared to type-I QW lasers, with spatially direct transitions and greater overlap between the electron and hole wavefunctions. Consequently, there has been renewed interest in incorporating type-I active regions within a cascading scheme to take advantage of the high optical gain associated with type-I QW lasers [29]-[32].

In the development of type-I devices with application grade performance, it is critical to determine the mechanisms which limit device performance and thermal stability. This is especially important as the operational wavelengths of type-I devices are pushed further into the mid-infrared where significant performance degradation has been observed. In this paper we report on the dominant loss and non-radiative recombination mechanisms affecting the operating characteristics of type-I GaInAsSb based lasers emitting at 2.3 $\mu \mathrm{m}, 2.6 \mu \mathrm{m}$ and $2.9 \mu \mathrm{m}$ wavelengths. Using additional results from the literature we present the wavelength dependence of the dominant efficiency limiting mechanisms at wavelengths between $2-3 \mu \mathrm{m}$.

\section{DEVICE STRUCTURES AND FABRICATION}

The laser hetrostructures were grown using solid source molecular beam epitaxy on an n-type doped GaSb substrate. In the $2.3 \mu \mathrm{m}$ structures the active region consisted of two $11 \mathrm{~nm}$ $\mathrm{Ga}_{0.63} \mathrm{InAs}_{0.11} \mathrm{Sb}$ quantum wells under $1.6 \%$ compressive strain. These were separated by $8 \mathrm{~nm} \mathrm{Al} 0_{0.33} \mathrm{GaAs}_{0.03} \mathrm{Sb}$ barriers. The active region was grown between $60 \mathrm{~nm} \quad \mathrm{Al}_{0.5} \mathrm{GaAs}_{0.04} \mathrm{Sb}$ confinement layers, followed by a $300 \mathrm{~nm} \mathrm{GaSb}$ waveguide and $2 \mu \mathrm{m} \mathrm{Al}{ }_{0.5} \mathrm{GaAs}_{0.04} \mathrm{Sb}$ cladding layers. The $2.6 \mu \mathrm{m}$ devices utilize a single $10 \mathrm{~nm} \mathrm{Ga} a_{0.57} \mathrm{InAs}_{0.14} \mathrm{Sb}$ quantum well under $1.7 \%$ compressive strain, grown between $10 \mathrm{~nm} \mathrm{GaSb}$ barriers. Surrounding the active region were undoped $\mathrm{Al}_{0.10} \mathrm{GaAs}_{0.01} \mathrm{Sb}$ waveguide layers and $2 \mu \mathrm{m}$ thick doped $\mathrm{Al}_{0.5} \mathrm{GaAs}_{0.04} \mathrm{Sb}$ cladding [33]. The active region of the $2.9 \mu \mathrm{m}$ devices was composed of three $10 \mathrm{~nm} \mathrm{Ga} 0.57 \mathrm{InAs}_{0.18} \mathrm{Sb}$ quantum wells under $1.4 \%$ compressive strain. The quantum wells were separated by $10 \mathrm{~nm} \mathrm{Al}{ }_{0.16} \mathrm{Ga}_{0.61} \mathrm{InAs}_{0.22} \mathrm{Sb}$ quinternary barriers. The active region was grown between $275 \mathrm{~nm} \mathrm{Al}_{0.15} \mathrm{GaAs}_{0.02} \mathrm{Sb}$ layers acting as a separate confinement layer and waveguide. This was followed by $2.5 \mu \mathrm{m} \mathrm{Al}_{0.5} \mathrm{GaAs}_{0.04} \mathrm{Sb}$ cladding [34].

A summary of device parameters are presented in Table 1.

TABLE 1

DEVICE PARAMETERS

\begin{tabular}{ccccc}
\hline \hline $\begin{array}{c}\text { DEVICE } \\
\text { WAVELENGTH }\end{array}$ & $\begin{array}{c}\text { NUMBER OF } \\
\text { QWs }\end{array}$ & $\begin{array}{c}\text { WidTH X LENGTH } \\
(\mu \mathrm{M})\end{array}$ & $\begin{array}{c}\Delta E_{\mathrm{C}} \\
(\mathrm{MEV})\end{array}$ & $\begin{array}{c}\Delta E_{\mathrm{V}} \\
(\mathrm{MEV}\end{array}$ \\
\hline $2.90 \mu \mathrm{m}$ & 3 & $30 \times 1700$ & 205 & 82 \\
$2.60 \mu \mathrm{m}$ & 1 & $60 \times 700$ & 160 & 54 \\
$2.30 \mu \mathrm{m}$ & 2 & $100 \times 650$ & 224 & 166 \\
\hline \hline
\end{tabular}

Number of QWs, ridge width and length as well as conduction band $\left(\Delta E_{\mathrm{c}}\right)$ and valence band $\left(\Delta E_{\mathrm{v}}\right)$ offsets for the three device structures investigated. The band offsets are defined between the band edges of the quantum well and barrier layers. 


\section{TEMPERATURE CHARACTERISTICS}

Applications for semiconductor lasers require low threshold current densities, $J_{t h}$ and crucially, stable performance over the operational temperature range. However, an exponential increase in the threshold current density is typically observed with increasing temperature [4]. The degradation in performance is generally attributed to the aggregate effect of non-radiative recombination, carrier leakage and optical loss processes such as IVBA. Since the recombination and loss mechanisms have a distinct dependence on temperature, the temperature dependence of the threshold current density can provide insight into the dominant efficiency limiting mechanisms.

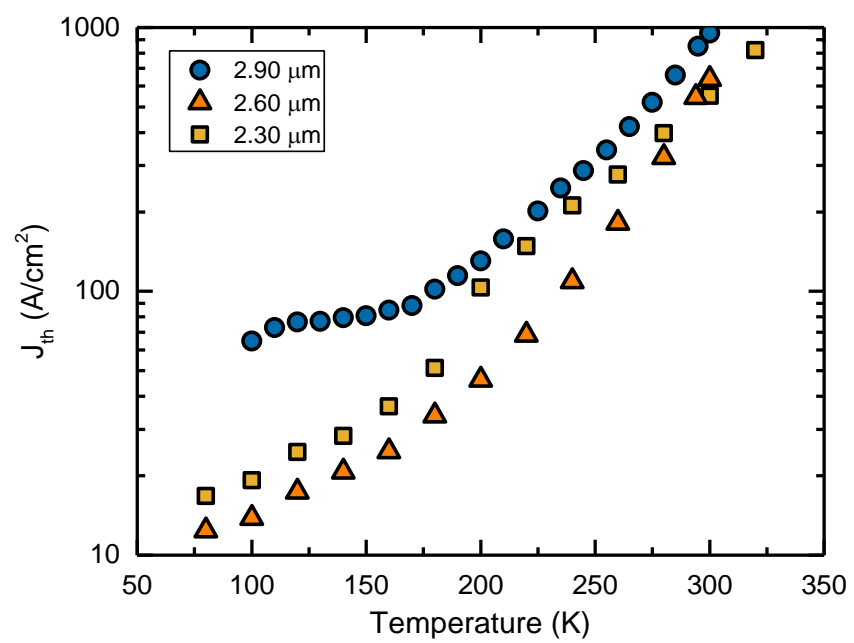

Fig. 1. Temperature dependence of the threshold current density for three typeI devices, operating at $2.3 \mu \mathrm{m}, 2.6 \mu \mathrm{m}$ and $2.9 \mu \mathrm{m}$. Approaching room temperature the threshold current density increases exponentially. A region of high temperature stability is observed in the $2.9 \mu \mathrm{m}$ between $110-160 \mathrm{~K}$.

The temperature characteristics of the three wavelength devices were measured using a static gas exchange liquidnitrogen $\left(\mathrm{N}_{2}\right)$ cryostat, between 80-300 K. To limit adverse heating effects, current was supplied in pulsed mode with a $0.05 \%$ duty cycle $(1 \mathrm{kHz}, 500 \mathrm{~ns}$ pulse width). Emission was measured with a liquid- $\mathrm{N}_{2}$ cooled InSb detector connected to a SR850 lock-in amplifier. The temperature dependence of the threshold current density for the three devices is presented in Fig 1. In each device the threshold current density increases exponentially approaching room temperature. Over a limited temperature range, the scale of this exponential increase may be described by the characteristic temperature, $T_{0}$, defined as [35];

$$
T_{0}=J_{t h}\left(\frac{d J_{t h}}{d T}\right)^{-1}
$$

Where $J_{\text {th }}$ is the threshold current density and $T$ is the temperature of the active region. A higher $T_{0}$ value is therefore desirable as it represents greater thermal stability of the threshold current density. Note that $T_{0}$ itself is, in general temperature dependent.

Between $80-160 \mathrm{~K}, T_{0}$ values of $103 \pm 6 \mathrm{~K}$ and $112 \pm 6 \mathrm{~K}$ were measured for the $2.3 \mu \mathrm{m}$ and $2.6 \mu \mathrm{m}$ devices. A value of $T_{0}$ for the $2.9 \mu \mathrm{m}$ device could not be uniquely defined over this temperature range due to the transient region of temperature stability between $110 \mathrm{~K}-160 \mathrm{~K}$, which from the definition in (1) leads to an infinite $T_{0}$ value. This behavior has been observed with other $\mathrm{Sb}$, dilute nitride and quantum dot-based lasers and may be attributed to compositional inhomogeneities in the grown layer [36]. Approaching room temperature, $T_{0}$ values of $56 \pm 6 \mathrm{~K}, 31 \pm 6 \mathrm{~K}$ and $42 \pm 1 \mathrm{~K}$ are measured for the 2.3 , 2.6 and $2.9 \mu \mathrm{m}$ devices respectively, between $260-300 \mathrm{~K}$. The dramatic increase in the temperature sensitivity (decreasing $\mathrm{T}_{0}$ ) with increasing temperature is consistent with the onset of a thermally activated loss or non-radiative recombination pathway. This behavior can be investigated further through a simple temperature dependence model for the radiative and non-radiative components of the threshold current density [37]. In the absence of carrier leakage and assuming equal electron and hole densities $(n=p)$ in the quantum wells, the threshold current density can be expressed phenomenologically as;

$$
J_{t h} \propto A n_{t h}+B n_{t h}^{2}+C n_{t h}^{3}
$$

Where the three terms account for monomolecular (defectrelated) recombination $\left(A n_{t h}\right)$, radiative recombination $\left(B n_{t h}^{2}\right)$ and Auger recombination $\left(\mathrm{Cn}_{t h}^{3}\right)$, respectively. Assuming the recombination coefficients are independent of carrier concentration, the temperature dependence of the radiative and Auger coefficients can be expressed as [38];

$$
\begin{gathered}
B \propto T^{-1} \\
C=C_{0} \exp \left(-E_{a} / k T\right)
\end{gathered}
$$

Where $E_{a}$ is the activation energy of an Auger process and $k$ is the Boltzmann constant. Additionally, for an ideal quantum well, the carrier density at threshold is expected to vary linearly with temperature. From these assumptions, expressions for the characteristic temperature due to radiative and Auger recombination can be derived $[39,40]$;

$$
\begin{gathered}
T_{0}\left(J_{\text {rad }}\right)=T \\
T_{0}\left(J_{\text {Aug }}\right)=\frac{T}{3+E_{a} / k T}
\end{gathered}
$$

The activation energy for the Auger process is expected to vary significantly between devices due to the strong wavelength dependence of the activation term. For simplicity, the activation energy may be temporarily neglected, reducing (6) to $T_{0}\left(J_{\text {Aug }}\right)=T / 3$. This expression therefore represents an upper limit on the characteristic temperature in a device dominated by a direct Auger process. The temperature dependence of $T_{0}$ for the three wavelength devices was calculated using 3-point differentiation and is presented in Fig 2. For the 2.3 and $2.6 \mu \mathrm{m}$ devices the characteristic temperature approximately follows the $T_{0} \approx T$ line at low temperatures. This behavior indicates 
that at low temperatures radiative recombination is the dominant recombination channel in these devices. This is maintained up to a breakpoint temperature, $T_{B}$, marking the onset of a thermally activated non-radiative channel [38]. For the $2.3 \mu \mathrm{m}$ device, the breakpoint temperature is measured as $T_{B} \sim 100 \mathrm{~K}$, while for the $2.6 \mu \mathrm{m}$ device, the breaking point temperature was slightly higher at $T_{B} \sim 120 \mathrm{~K}$. The low temperature behavior of $T_{0}$ in the $2.9 \mu \mathrm{m}$ device is again obscured by the region of high stability in the threshold current and so is omitted from this analysis. Approaching room temperature the characteristic temperature of all three devices approaches and subsequently falls below the $\mathrm{T} / 3$ line.

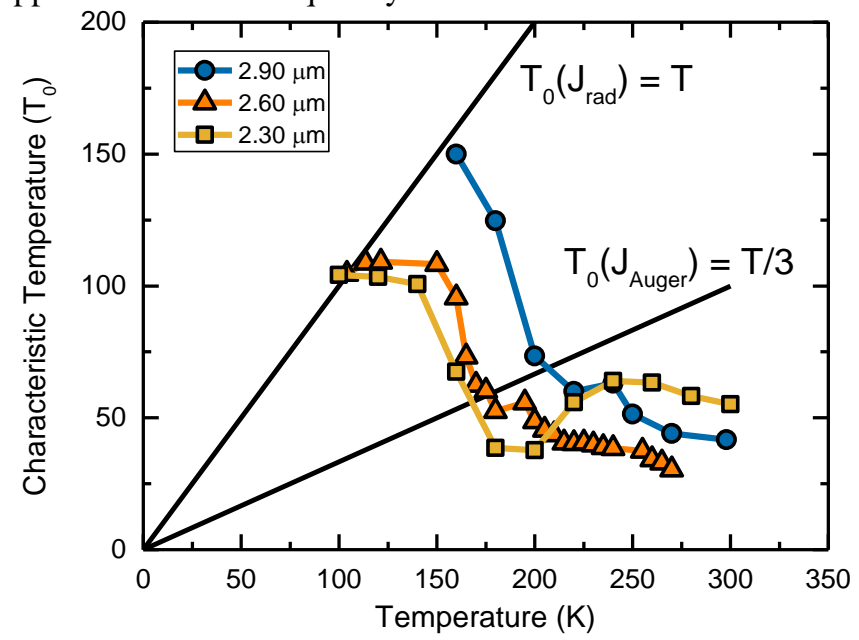

Fig. 2. The temperature dependence of the characteristic temperature. At low cryogenic temperatures the characteristic temperature is consistent with a device dominated by radiative recombination. This behavior is maintained up to a break point temperature, marking the onset of a thermal activated nonradiative channel.

While this analysis confirms the activation of a non-radiative channel, it is clear that a more sophisticated model is required in order to properly account for the high temperature sensitivity at room temperature. Inclusion of an Auger activation term would predict a significantly lower value for $T_{0}$ and may account for the discrepancy. In the following section contributions of the direct Auger mechanisms to the threshold current density are determined, and the activation energy terms discussed in more detail. In addition, there are other important mechanisms that this simple temperature model omits, these include carrier leakage and optically losses, both of which act to reduce $T_{0}$ further [41].

\section{Hydrostatic PRESSURE}

Hydrostatic pressure measurements are uniquely capable of decoupling the effect of different recombination channels on the threshold current density. The application of hydrostatic pressure compresses the semiconductor device while preserving crystal symmetries. This reversible procedure increases the direct bandgap energy in III-V semiconductor devices, typically at a rate of approximately $10 \mathrm{meV} / \mathrm{kbar}$ [42]. The dominant recombination mechanisms at room temperature can then be determined by measuring the pressure dependence of the threshold current density. The pressure dependent characteristics of different recombination pathways are illustrated in Fig 3.

Carrier Leakage: III-V semiconductors and their alloys are all observed to have broadly similar pressure coefficients. The conduction and valence band offsets that determine the amount of carrier leakage are therefore not expected to change significantly under hydrostatic pressure. This prediction has been substantiated by a number of experimental [43]-[45] and theoretical studies [46], [47]. Over the hydrostatic pressure range used in this work, carrier leakage and its contribution to threshold are therefore expected to have only a weak pressure dependence.

Radiative Recombination: In an ideal quantum well which operates close to transparency, the radiative current scales with bandgap according to, $J_{\text {rad }} \propto E_{g}^{2}$. Experimental work under hydrostatic pressure has shown this relation to be a reasonable approximation [48], [49].

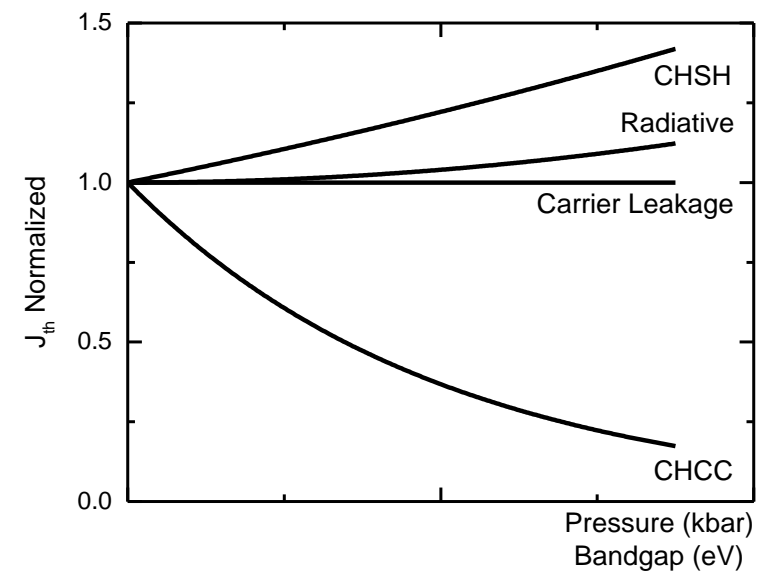

Fig. 3. Pressure dependent characteristics for several important recombination channel in mid-infrared devices.

Auger Recombination: as stated in (4) the Auger coefficient can be expressed as $C=C_{0} \exp \left(-E_{a} / k T\right)$. The activation energy associated with a particular Auger process is a consequence of energy and momentum conservation which limits the electron-hole distributions which can participate in an Auger process. Within the isotropic and parabolic band approximation, the activation energies can be derived analytically through Boltzmann statistics. For the direct CHCC and CHSH Auger processes, the activation energies are [50];

$$
\begin{aligned}
& E_{a}^{C H C C} \approx \frac{m_{c}}{m_{c}+m_{h}} E_{g} \\
& E_{a}^{C H S H} \approx \Delta_{S O}-E_{g}, \quad \Delta_{S O}>E_{g}
\end{aligned}
$$

Where $E_{g}$ is the bandgap and $\Delta_{S O}$ is the magnitude of the spin-orbit split-off energy. For quantum wells, $m_{c}$ and $m_{h}$ refer to the in-plane effective mass of the conduction and heavy hole bands respectively. The activation energy expressed in (8) strictly applies in the regime where the energy separation of the spin-orbit split-off band is greater than the bandgap energy, as is the case in these GaInAsSb devices. 
The activation energies predict that in a device dominated by the CHCC Auger process, the threshold current density should decrease with an approximately exponentially dependence under pressure. Since in these devices, the bandgap energy is less than the spin-orbit split-off energy, the CHSH component is expected to increase exponentially as the bandgap approaches resonance with the split-off band.

In this work the room temperature threshold current density was measured over a hydrostatic pressure range of $0-8 \mathrm{kbar}$. Pressure was applied using a helium gas compressor system (UniPress U11) and emission was measured using a liquid $\mathrm{N}_{2}$ cooled InSb detector connected to a SR830 lock-in amplifier. The threshold current density of the three wavelength devices measured under hydrostatic pressure is presented in fig 4 . The exponential decrease in the threshold current density clearly identifies the CHCC Auger mechanism as the dominant efficiency limiting process in each of these devices. While carrier leakage is expected to have some effect on the threshold current density, its contribution must be secondary to that of CHCC recombination in order to explain the strong pressure dependence of the threshold current density.

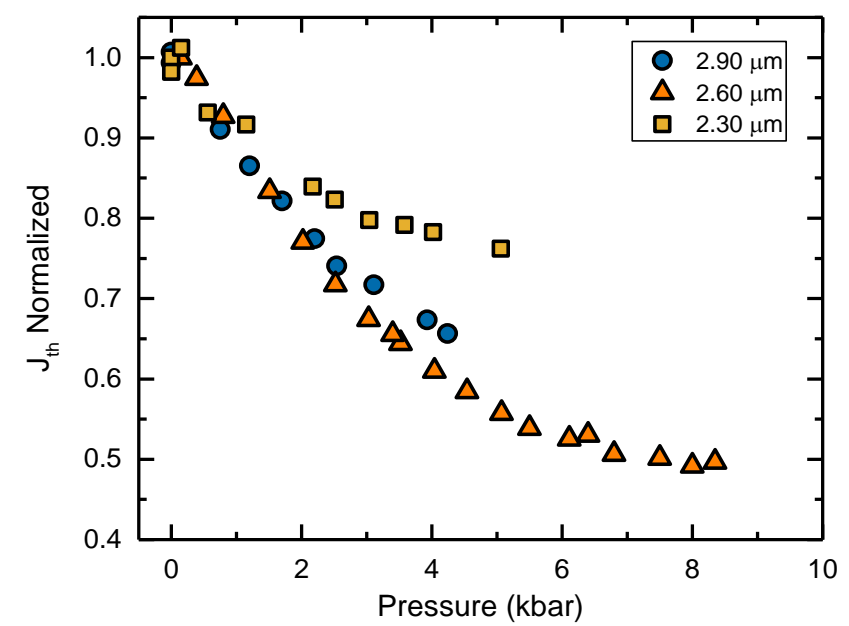

Fig. 4. Normalized threshold current density as a function of pressure. In each wavelength device the threshold current density decreases exponentially. This behavior can be explained by the dominance of the CHCC Auger process at room temperature.

Hydrostatic pressure measurements on type-I GaInAsSb lasers operating at $2.37 \mu \mathrm{m}$ and $2.11 \mu \mathrm{m}$ have been reported in the literature, in each case identifying an Auger process as the dominant non-radiative recombination pathway [51], [52]. The hydrostatic measurements reported here, supplemented with the literature measurements, offer a unique opportunity to analyze the wavelength dependence of the efficiency limiting mechanisms in the $2-3 \mu \mathrm{m}$ range.

In order to determine the effect of pressure on the bandgap, the emission spectrum for the 2.3 and $2.6 \mu \mathrm{m}$ lasers were measured under pressure using Fourier Transform Infrared Spectroscopy (FTIR). Over the pressure range of the measurements, the lasing peak shift under pressure gave linear pressure coefficients of 10.6 and $10.2 \mathrm{meV} / \mathrm{kbar}$ for the 2.3 and $2.6 \mu \mathrm{m}$ devices respectively. These values are in good agreement with pressure coefficients reported for the $2.11 \mu \mathrm{m}$ devices. For the purpose of this analysis the pressure coefficient was taken as $10.4 \mathrm{meV} / \mathrm{kbar}$, an average of the available values.

Isolating the non-radiative component of the threshold current density requires extraction of the radiative recombination component of the threshold current density. The radiative component can be determined by measuring the integrated spontaneous emission as a function of temperature (not reported here), and assuming that at low temperatures the threshold current density is dominated by radiative recombination. This assumption is supported by the low temperature behavior of the characteristic temperature in the 2.6 $\mu \mathrm{m}$ and $2.9 \mu \mathrm{m}$ devices. Normalizing the low temperature integrated spontaneous emission to the threshold current density, the radiative component of the threshold current at room temperature can then be determined. Using this approach, the radiative component of the threshold current density was estimated to be in the range of 5\%-20\% in the devices studied, clearly highlighting the importance of non-radiative recombination. Using $J_{\text {rad }} \propto E_{g}^{2}$, the radiative component of the threshold current density can thus be extracted from measurements under hydrostatic pressure.

The different wavelength devices used in this analysis vary significantly in their composition, strain and device structure. These differences are reflected in their threshold current densities. It is therefore necessary to normalize the nonradiative threshold current density to observe the underlying trend. To normalize these results, the non-radiative threshold current density is plotted as a function of lasing energy( bandgap). Each device is then normalized relative to the $2.9 \mu \mathrm{m}$ device in order to produce a smooth dependence with lasing energy. The result of this normalization procedure is presented in fig 5. It is notable that despite considerable variation between device designs that a clear bandgap dependence in the non-radiative current between $2-3 \mu \mathrm{m}$ is observed. This indicates that we are observing the variation of an effect that is primarily dependent on the electronic band structure, which is changing with pressure, and not on the physical structure of the laser. The later determines the absolute threshold current but is essentially independent of pressure.

Two important regimes are evident in fig 5 . From $0.4 \mathrm{eV}$, the non-radiative current decays approximately exponentially with bandgap up to a turning point around $0.6 \mathrm{eV}$. For energies above this point, the non-radiative threshold current density then increases with bandgap. The strong wavelength dependence of the non-radiative current, in conjunction with the temperature characteristics, clearly identifies an Auger recombination mechanism as the dominant recombination channel at room temperature. The transitional behavior of the non-radiative threshold current is consistent with the reduced CHCC Auger rate and the activation of the CHSH Auger process around 0.6 $\mathrm{eV}$, as the bandgap approaches resonance with the spin-orbit split-off band. In order to illustrate this transitional effect, the non-radiative component of the threshold current density is modelled assuming the non-radiative current is determined only by direct $\mathrm{CHCC}$ and $\mathrm{CHSH}$ recombination. In a regime dominated by the $\mathrm{CHCC}$ and $\mathrm{CHSH}$ processes, assuming Boltzmann statistics and equal electron and hole densities, the 
Auger coefficient can be expressed in the following form;

$$
C=C_{0, C H C C} \exp \left(-E_{a}^{C H C C} / k T\right)+C_{0, C H S H}\left(-E_{a}^{C H S H} / k T\right)
$$

TABLE I

MATERIAL PARAMETERS

\begin{tabular}{cc}
\hline \hline Parameter & Value \\
\hline $\mathrm{m}_{\mathrm{c}}$ & $0.038 \mathrm{~m}_{0}$ \\
$m_{h}$ & $0.252 \mathrm{~m}_{0}$ \\
$m_{l}$ & $0.049 \mathrm{~m}_{0}$ \\
$E_{g}$ & $0.477 \mathrm{eV}$ \\
$E_{s o}$ & $0.700 \mathrm{eV}$ \\
\hline \hline
\end{tabular}

List of the material parameters used to generate the fit for the non-radiative component of the threshold current density. The values are estimates for unstrained $\mathrm{Ga}_{0.33} \mathrm{InAs}_{0.03} \mathrm{Sb}$ which is the composition in quantum wells of the $2.3 \mu \mathrm{m}$ device.

Where the activation energies, $E_{a}$ are defined in (7) and (8).

It is also reasonable to assume that over the range of the hydrostatic pressure measurements the threshold carrier density does not change significantly, as previously shown theoretically [53]. The Auger coefficient expressed in (9) therefore represents the Auger current density, provided the electron and hole carrier densities can be taken as equal in the quantum well. For the purpose of the model, the activation energies were calculated using materials parameters for the GaInAsSb quantum well of the $2.3 \mu \mathrm{m}$ device. The material parameters used for the fit are listed in Table 1 [54].

The change in the bandgap energy will also modify the effective mass of the conduction band, $m_{c}$, and light hole, $m_{l}$. This effect can be understood through k.p theory where the effective mass scales in the same proportion as the bandgap [55]. Finally, in order to generate the fit, it was assumed that the bandgap dependence of the Auger processes are primarily determined by the exponential term. The $C_{0}$ coefficients for the two Auger processes were therefore varied independently as single valued fitting parameters. The assumptions used to construct this simple model clearly have limited validity. 1) the Boltzmann approximation is strictly legitimate only for low carrier concentrations far from threshold. 2) in compressively strained quantum well devices the bands are highly nonparabolic and anisotropic. Consequently, the effective mass terms that determine the Auger activation energies then become poorly defined. 3 ) the $C_{0}$ coefficients are assumed to be single valued, neglecting any bandgap dependence. Despite these approximations, the model can provide useful insight into the qualitative behavior of the $\mathrm{CHCC}$ and $\mathrm{CHSH}$ processes, accounting for the wavelength dependence observed in the nonradiative threshold current density. Within the constraints of the simple model proposed here some effort can be made to include the non-parabolicity of the band structure. Under compressive strain, the mass of the valence band at the zone center tends to that of the unstrained light hole mass [56]. However, further out in k-space where the Auger processes typically occur, the valence band takes on an increasingly heavy character. To account for this, the fit is applied separately using the light and heavy hole masses of the unstrained quantum well material. These two extreme cases bound the shaded region in Fig 5 .

In Fig 5, the qualitative behavior of the experimental data is reproduced irrespective of the valence band mass used to generate the fit. Significantly, these results capture the regimes where the $\mathrm{CHCC}$ and $\mathrm{CHSH}$ mechanisms represent the dominant efficiency limiting mechanisms. The results indicate the dominance of the CHCC process above $2 \mu \mathrm{m}$ and show clearly the rapidly increasing CHCC recombination rate, which has been so detrimental to device performance in mid-infrared devices. The excellent fit between experiment and the simple theory over this wavelength range may reflect the fact that the CHCC process involves excitation of electrons to at least the band gap energy away from the band edge. The recombining electron then involves a hole which has the same large momentum and so is likely to be in the Boltzmann tail of the carrier distribution. At wavelengths below $2 \mu \mathrm{m}$, although the band gap approaches the spin-orbit split-off energy, the onset of the CHSH process will again involve the most energetic carriers which are to be found in the Boltzmann tail. As the wavelength shortens the $\mathrm{CHSH}$ process can occur with more vertical transitions close to the zone center. This results in a significant enhancement of the Auger recombination rate and is reflected in the increasing threshold current density at shorter wavelengths, as has indeed been observed in $1.55 \mu \mathrm{m}$ telecommunications lasers [57].

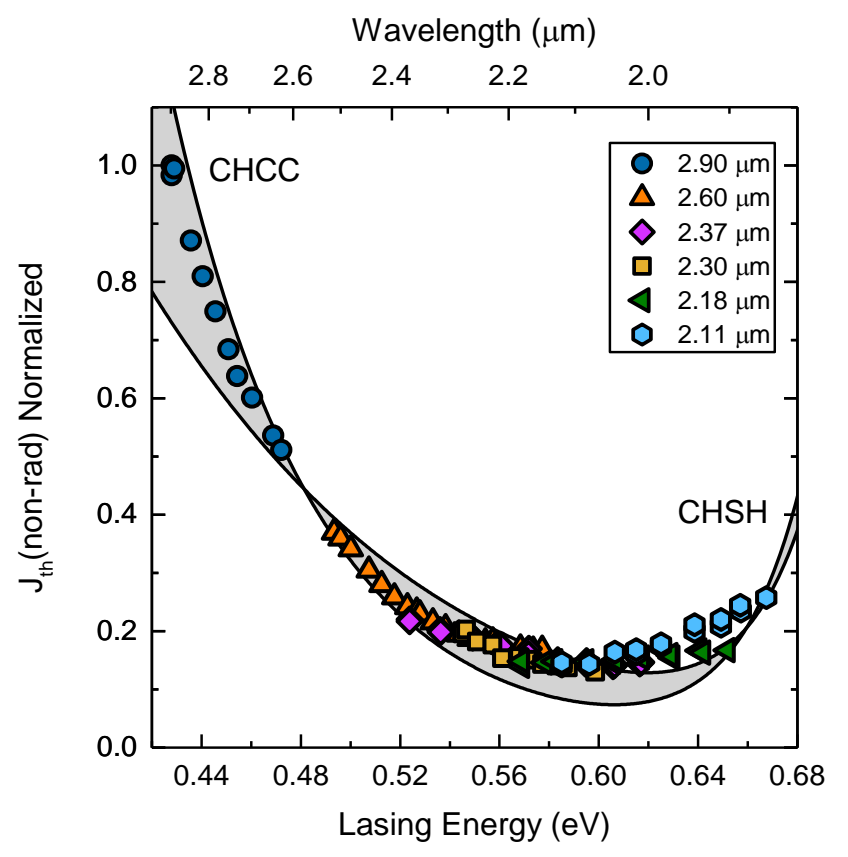

Fig. 5. Normalized non-radiative threshold current as a function of lasing energy. The fit was performed using material parameters from the $2.3 \mu \mathrm{m}$ device with a spin-orbit split-off energy of $0.70 \mathrm{eV}$. The shaded area represents the region bounded by the fits generated using a light or heavy hole in-plane mass. 


\section{CONCLUSION}

In this study we report on the efficiency limiting mechanisms of type-I GaInAsSb-based lasers operating between 2-3 $\mu \mathrm{m}$. The dominant recombination channels responsible for the high temperature sensitivity was investigated using three midinfrared devices operating at $2.9 \mu \mathrm{m}, 2.6 \mu \mathrm{m}$ and $2.3 \mu \mathrm{m}$. The temperature dependence revealed that at very low (cryogenic) temperatures radiative recombination dominates the threshold current density. At higher temperatures, the characteristic temperature decreases sharply, marking the onset of a thermally activated recombination pathway. To determine the nonradiative mechanism that dominates the threshold and determines the high temperature sensitivity at room temperature, the threshold current density was measured under hydrostatic pressure. Hydrostatic pressure reversibly modifies the bandgap energy independently of temperature. Measurements under hydrostatic pressure clearly identifies the CHCC Auger process as the dominant efficiency limiting mechanism, at room temperature, in these devices. To establish the wavelength dependence of the dominant non-radiative channels these results are supplemented with high pressure measurements from three other type-I GaInAsSb lasers. After removing the radiative component of the threshold current density the non-radiative component in each device is normalized and found to produce a smooth wavelength dependence. Despite considerable variation in material parameters and device processing, the underlying wavelength dependence of the non-radiative current was consistent between devices. These results motivated modelling of the non-radiative current across the 2-3 $\mu \mathrm{m}$ wavelength range. A simple model using the $\mathrm{CHCC}$ and $\mathrm{CHSH}$ Auger mechanisms reproduces the qualitative behavior of the experimental data. The results reveal two important regimes. Between 2-3 $\mu \mathrm{m}$, the CHCC Auger process dominates the non-radiative threshold current. This is reflected in the exponential increase of the normalized nonradiative current with increasing wavelength and explains the severe performance degradation observed in long wavelength type-I devices. At wavelengths around $2 \mu \mathrm{m}$, the bandgap energy begins to approach resonance with the spin-orbit splitoff energy, and the $\mathrm{CHSH}$ process becomes activated. At wavelengths below $2 \mu \mathrm{m}$ the $\mathrm{CHSH}$ Auger process consequently becomes the dominant efficiency limiting mechanism, as previously seen in $1.55 \mu \mathrm{m}$ lasers.

\section{REFERENCES}

[1] E. Geerlings, M. Rattunde, J. Schmitz, G. Kaufel, H. Zappe, and J. Wagner, "Widely tunable GaSb-based external cavity diode laser emitting around $2.3 \mu \mathrm{m}$," IEEE Photonics Technol. Lett., vol. 18, no. 18, pp. 1913-1915, 2006.

[2] A. Gonzalvez, S. Garrigues, M. de la Guardia, and S. Armenta, "The ways to the trace level analysis in infrared spectroscopy," Anal. Methods, vol. 3, no. 1, pp. 43-52, 2011.

[3] A. Joullié and P. Christol, "GaSb-based mid-infrared 2-5 $\mu \mathrm{m}$ laser diodes," Comptes Rendus Phys., vol. 4, no. 6, pp. 621-637, 2003.

[4] S. D. Sifferman, H. P. Nair, R. Salas, N. T. Sheehan, S. J. Maddox, A. M. Crook, and S. R. Bank, "Highly Strained Mid-Infrared Type-I Diode Lasers on GaSb," IEEE J. Sel. Top. Quantum Electron., vol. 21, no. 6, 2015.

[5] J. G. Kim, L. Shterengas, R. U. Martinelli, G. L. Belenky, D. Z. Garbuzov, and W. K. Chan, "Room-temperature $2.5 \mu \mathrm{m}$ InGaAsSb/AlGaAsSb diode lasers emitting $1 \mathrm{~W}$ continuous waves," Appl. Phys. Lett., vol. 81, no. 17, pp. 3146-3148, 2002.
J. G. Kim, L. Shterengas, R. U. Martinelli, and G. L. Belenky, "High-power room-temperature continuous wave operation of type-I $\mathrm{In}(\mathrm{Al}) \mathrm{GaAsSb} / \mathrm{GaSb}$ diode lasers at wavelengths greater than 2.5 um," Proc. SPIE Int. Soc. Opt. Eng. 2004; 5365(1) 240 9, vol. 5365, pp. 240-249, 2004.

[7] T. Hosoda, G. Kipshidze, L. Shterengas, and G. Belenky, "Diode lasers emitting near $3.44 \mu \mathrm{m}$ in continuous-wave regime at $300 \mathrm{~K}$," Electron. Lett., vol. 46, no. 21, pp. 1455-1457, 2010.

[8] K. Vizbaras and M.-C. Amann, "Room-temperature $3.73 \mu \mathrm{m} \mathrm{GaSb}$ based type-I quantum-well lasers with quinternary barriers," Semicond. Sci. Technol., vol. 27, no. 3, pp. 1-4, 2012.

[9] K. Vizbaras and M.-C. Amann, "3.6 $\mu \mathrm{m}$ GaSb-based type-I lasers with quinternary barriers, operating at room temperature," Electron. Lett., vol. 47, no. 17, p. 980981, 2011.

[10] R. G. Bedford, G. Triplett, D. H. Tomich, S. W. Koch, J. Moloney, and J. Hader, "Reduced auger recombination in mid-infrared semiconductor lasers," J. Appl. Phys., vol. 110, no. 7, pp. 73108-173108-6, 2011

[11] K. S. Gadedjisso-Tossou, S. Belahsene, M. A. Mohou, E. Tournié, and Y. Rouillard, "Recombination channels in 2.4-3.2 $\mu \mathrm{m}$ GaInAsSb quantum-well lasers," Semicond. Sci. Technol., vol. 28, no. 1, pp. 15015-1-15015-6, 2013

[12] S. Anikeev, D. Donetsky, G. Belenky, S. Luryi, C. A. Wang, J. M. Borrego, and G. Nichols, "Measurement of the Auger recombination rate in p-type $0.54 \mathrm{eV}$ GaInAsSb by time- resolved photoluminescence," Appl. Phys. Lett., vol. 83, no. 16, pp. 33173319, 2003.

[13] A. R. Adams, M. Asada, Y. Suematsu, and S. Arai, "The Temperature Dependence of the Efficiency and Threshold Current of In1- xGaxAsyP1-y Lasers Related to Intervalence Band Absorption," Jpn. J. Appl. Phys., vol. 19, no. 10, pp. L621-624, 1980.

[14] A. B. Ikyo, I. P. Marko, K. Hild, A. R. Adams, S. Arafin, M.-C. Amann, and S. J. Sweeney, "Temperature stable mid-infrared GaInAsSb/GaSb Vertical Cavity Surface Emitting Lasers (VCSELs).," Sci. Rep., vol. 6, p. 19595, Jan. 2016.

[15] J. Angellier, D. Barat, G. Boissier, F. Chevier, P. Grech, and Y. Rouillard, "Toward an $\mathrm{AlGaAsSb} / \mathrm{GaInAsSb} / \mathrm{GaSb}$ laser emitting beyond $3 \mu \mathrm{m}$," Proc. SPIE, vol. 6485B, p. 64850B-1-64850B-11, 2007.

[16] L. Shterengas, G. L. Belenky, J. G. Kim, and R. U. Martinelli, "Design of high-power room-temperature continuous-wave GaSbbased type-I quantum-well lasers with $>2.5 \mu \mathrm{m}$," Semicond. Sci. Technol., vol. 19, no. 5, pp. 655-658, 2004.

[17] J. Chen, D. V Donetski, L. Shterengas, M. V Kisin, G. Kipshidze, and G. L. Belenky, "Effect of Quantum Well Compressive Strain Above 1\% On Differential Gain and Threshold Current Density of Type-I GaSb-based Diode Lasers," IEEE J. Quantum Electron., vol. 44, no. 12, pp. 1204-1210, 2008.

[18] R. Liang, J. Chen, G. Kipshidze, D. Westerfeld, L. Shterengas, and G. Belenky, "High-Power 2.2 um Diode Lasers With Heavily Strained Active Region," IEEE Photonics Technol. Lett., vol. 23, no. 10 , pp. $603-605,2011$.

[19] T. Hosoda, G. Kipshidze, G. Tsvid, L. Shterengas, and G. Belenky, "Type-I GaSb-Based Laser Diodes Operating in 3.1 to $3.3 \mu \mathrm{m}$ Wavelength Range," IEEE Photonics Technol. Lett., vol. 22, no. 10, pp. 718-720, 2010.

[20] J. Chen, T. Hosoda, G. Tsvid, R. Liang, D. Westerfeld, G. Kipshidze, L. Shterengas, and G. Belenky, "Type-I GaSb based diode lasers operating at room temperature in 2 to $3.5 \mu \mathrm{m}$ spectral region," Proc. SPIE, vol. 7686, p. 76860S-1-76860S-5, 2010

[21] M. Razeghi, N. Bandyopadhyay, Y. Bai, Q. Lu, and S. Slivken, "Recent advances in mid infrared (3-5 $\mu \mathrm{m})$ Quantum Cascade Lasers," Opt. Mater. Express, vol. 3, no. 11, pp. 1872-1884, 2013.

[22] D. Botez, C.-C. Chang, and L. J. Mawst, "Temperature sensitivity of the electro-optical characteristics for mid-infrared $(\lambda=3-16 \mu \mathrm{m})$ emitting quantum cascade lasers," J. Phys. D. Appl. Phys., vol. 49, no. 4, pp. 1-33, 2016.

[23] A. N. Baranov and R. Teissier, "Quantum Cascade Lasers in the InAs/AlSb Material System," IEEE J. Sel. Top. Quantum Electron., vol. 21, no. 6, pp. 1-12, 2015.

[24] O. Cathabard, R. Teissier, J. Devenson, J. C. Moreno, and A. N. Baranov, "Quantum cascade lasers emitting near $2.6 \mu \mathrm{m}, "$ Appl. Phys. Lett., vol. 96, no. 14, pp. 32-35, 2010.

[25] N. Bandyopadhyay, Y. Bai, S. Tsao, S. Nida, S. Slivken, and M. 
Razeghi, "Room temperature continuous wave operation of $\lambda \sim 3-$ $3.2 \mu \mathrm{m}$ quantum cascade lasers," Appl. Phys. Lett., vol. 101, no. 2012, p. 241110, 2012.

[26] I. P. Marko, A. R. Adams, S. J. Sweeney, R. Teissier, A. N. Baranov, and S. Tomić, "Evidence of carrier leakage into the Lvalley in InAs-based quantum cascade lasers under high hydrostatic pressure," Phys. Status Solidi Basic Res., vol. 246, no. 3, pp. 512$515,2009$.

[27] J. Scheuermann, R. Weih, M. Von Edlinger, L. Nähle, M. Fischer, J. Koeth, M. Kamp, and S. Höfling, "Single-mode interband cascade lasers emitting below $2.8 \mu \mathrm{m}$," Appl. Phys. Lett., vol. 106, no. 16, pp. $1-5,2015$.

[28] G. G. Zegrya and A. D. Andreev, "Mechanism of suppression of Auger recombination processes in type-II heterostructures," Appl. Phys. Lett., vol. 67, no. 18, pp. 2681-2683, 1995.

[29] L. Shterengas, R. Liang, G. Kipshidze, T. Hosoda, S. Suchalkin, and G. Belenky, "Type-I quantum well cascade diode lasers emitting near 3 um," Appl. Phys. Lett., vol. 103, no. 2014, pp. 1-5, 2013.

[30] L. Shterengas, R. Liang, G. Kipshidze, T. Hosoda, G. Belenky, S. S. Bowman, and R. L. Tober, "Cascade type-I quantum well diode lasers emitting $960 \mathrm{~mW}$ near $3 \mu \mathrm{m}$," Appl. Phys. Lett., vol. 105, no. 16, pp. 161112-1-161112-4, 2014.

[31] Y. Jiang, L. Li, R. Q. Yang, J. A. Gupta, G. C. Aers, E. Dupont, J.M. Baribeau, X. Wu, and M. B. Johnson, "Type-I interband cascade lasers near 3.2 m," Appl. Phys. Lett., vol. 106, pp. 0-41117$141117-4,2015$.

[32] L. Shterengas, G. Kipshidze, T. Hosoda, M. Wang, T. Feng, and G. Belenky, "Cascade Type-I Quantum Well GaSb-Based Diode Lasers," Photonics, vol. 3, no. 27, pp. 1-10, 2016.

[33] K. Kashani-Shirazi, K. Vizbaras, A. Bachmann, S. Arafin, and M.C. Amann, "Low-Threshold Strained Quantum-Well GaSb-Based Lasers Emitting in the 2.5-2.7- $\mu \mathrm{m}$ Wavelength Range," IEEE Photonics Technol. Lett., vol. 21, no. 16, pp. 1106-1108, 2009.

[34] K. Vizbaras, A. Andrejew, A. Vizbaras, C. Grasse, S. Arafin, and M.-C. Amann, "Low-threshold $3 \mu \mathrm{m}$ GaInAsSb/AlGaInAsSb quantum-well lasers operating in continuous-wave up to $64 \mathrm{C}$," IPRM 2011 23rd Int. Conf. Indium Phosphide Relat. Mater., pp. 14, 2011.

[35] T. Higashi, T. Yamamoto, S. Ogita, and M. Kobayashi, "Experimental analysis of characteristic temperature in quantumwell semiconductor lasers," IEEE J. Sel. Top. Quantum Electron., vol. 3, no. 2, pp. 513-521, 1997.

[36] R. Fehse, S. Tomić, A. R. Adams, S. J. Sweeney, E. P. O'Reilly, A. Andreev, and H. Riechert, "A quantitative study of radiative, auger, and defect related recombination processes in 1.3- $\mu \mathrm{m}$ GaInNAsbased quantum-well lasers," IEEE J. Sel. Top. Quantum Electron., vol. 8, no. 4, pp. 801-810, 2002.

[37] S. J. Sweeney, S. R. Jin, C. N. Ahmad, A. R. Adams, and B. N. Murdin, "Carrier recombination processes in $1.3 \mu \mathrm{m}$ and $1.5 \mu \mathrm{m}$ InGaAs(P)-based lasers at cryogenic temperatures and high pressures," Phys. Status Solidi, vol. 241, no. 14, pp. 3399-3404, 2004.

[38] S. J. Sweeney, A. Phillips, A. R. Adams, E. P. O'Reilly, and P. J. A. Thijs, "The effect of temperature dependent processes on the performance of $1.5-\mu \mathrm{m}$ compressively strained InGaAs (P) MQW semiconductor diode lasers," Photonics Technol. Lett., vol. 10, no. 8, pp. 1076-1078, 1998

[39] N. Tansu and L. J. Mawst, "Design analysis of 1550-nm GaAsSb(In)GaAsN type-II quantum-well laser active regions," IEEE J. Quantum Electron., vol. 39, no. 10, pp. 1205-1210, 2003.

[40] E. P. O'Reilly and M. Silver, "Temperature sensitivity and high temperature operation of long wavelength semiconductor lasers," Appl. Phys. Lett., vol. 63, no. 24, pp. 3318-3320, 1993.

[41] S. J. Sweeney and P. Thijs, "Origin of the High Temperature Performance Degradation of $1.5 \mu \mathrm{m} \operatorname{InGaAs}(\mathrm{P}) / \mathrm{InP}$ Quantum Well Lasers," IEEE LEOS, vol. 2, pp. 977-978, 2003.

[42] S. Wei and A. Zunger, "Predicted band-gap pressure coefficients of all diamond and zinc-blende semiconductors: Chemical trends (Wei)," Phys. Rev. B, vol. 60, no. 8, pp. 5404-5411, 1999.

[43] H. M. Cheong, J. H. Burnett, W. Paul, P. F. Hopkins, and A. C. Gossard, "Hydrostatic-pressure dependence of band offsets in GaAs/AlxGa1-xAs hetrostructures," Phys. Rev. B, vol. 49, no. 15, p. 10 444-10 449, 1994.

[44] J. D. Lambkin, D. J. Dunstan, E. P. O. Reilly, and B. R. Butler, "The Pressure Dependence of the Band Offsets in a GaInAs/InP
Multiple Quantum Well Structure," J. Cryst. Growth, vol. 93, pp. 323-328, 1988.

[45] J. D. Lambkin, A. R. Adams, D. J. Dunstan, P. Dawson, and C. T. Foxon, "Pressure dependence of the valence-band discontinuity in GaAs/AlAs and GaAs/AlxGa1-xAs quantum well structures," Phys. Rev. B, vol. 39, no. 8, pp. 5546-5549, 1989.

[46] C. V. De Walle and R. M. Martin, "Theoretical study of band offsets at semiconductor interfaces," Phys. Rev. B, vol. 35, no. 15, pp. 8154-8165, 1987.

[47] H. Ünlü, "Tight binding modeling of heterojunction band offsets as a function of pressure and composition," Phys. Status Solidi Basic Res., vol. 223, no. 1, pp. 195-204, 2001.

[48] K. Hild, S. J. Sweeney, I. P. Marko, S. R. Jin, S. R. Johnson, S. a. Chaparro, S. Yu, and Y.-H. Zhang, "Temperature and pressure dependence of carrier recombination processes in $\mathrm{GaAsSb} / \mathrm{GaAs}$ quantum well lasers," Phys. Status Solidi, vol. 244, no. 1, pp. 197202, 2007.

[49] N. F. Massé, A. R. Adams, and S. J. Sweeney, "Experimental determination of the band gap dependence of Auger recombination in InGaAs/InP multiple quantum well lasers at room temperature," Appl. Phys. Lett., vol. 90, no. 16, pp. 161113-1-161113-3, 2007.

[50] H. K. Choi and G. W. Turner, "Antimonide-based strained quantum-well diode lasers," Phys. Scr., vol. T69, pp. 17-25, 1997.

[51] K. O’Brien, S. J. Sweeney, A. R. Adams, B. N. Murdin, A. Salhi, Y Rouillard, and A. Joullié, "Recombination processes in midinfrared InGaAsSb diode lasers emitting at $2.37 \mu \mathrm{m}$," Appl. Phys. Lett., vol. 89, no. 51104, pp. 51104-1-51104-3, 2006.

[52] K. O'Brien, S. J. Sweeney, A. R. Adams, S. R. Jin, C. N. Ahmad, B N. Murdin, A. Salhi, Y. Rouillard, and A. Joullié, "Carrier recombination mechanisms in mid-infrared GaInAsSb quantum well lasers," Phys. Status Solidi, vol. 207, no. 1, pp. 203-207, 2007.

[53] M. Silver, E. P. O'Reilly, and A. R. Adams, "Determination of the wavelength dependence of auger recombination in long-wavelength quantum-well semiconductor lasers using hydrostatic pressure," IEEE J. Quantum Electron., vol. 33, no. 9, pp. 1557-1566, 1997.

[54] I. Vurgaftman, J. R. Meyer, and L. R. Ram-Mohan, "Band parameters for III-V compound semiconductors and their alloys," $J$. Appl. Phys., vol. 89, no. 11 I, pp. 5815-5875, 2001.

[55] M. Silver, E. P. O'Reilly, B. Gonul, A. F. Phillips, S. J. Sweeney, and P. J. A. Thijs, "Hydrostatic Pressure Dependence of the Threshold Current in 1.5 pm Strained Quantum Well Lasers," Phys. Status Solidi, vol. 198, no. 381, pp. 381-388, 1996.

[56] A. R. Adams, "Strained-layer quantum-well lasers," IEEE J. Sel. Top. Quantum Electron., vol. 17, no. 5, pp. 1364-1373, 2011.

[57] A. F. Phillips, S. J. Sweeney, A. R. Adams, and P. J. A. Thijs, "The Temperature Dependence of 1.3- and 1.5- $\mu \mathrm{m}$ Compressively Strained $\operatorname{InGaAs}(\mathrm{P}) \mathrm{MQW}$ Semiconductor Lasers," IEEE J. selcted Top. Quantum Electron., vol. 5, no. 3, pp. 401-412, 1999.

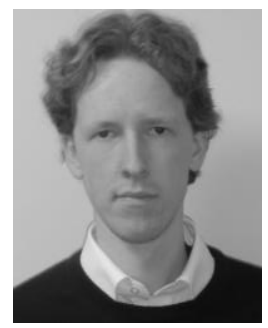

Timothy D. Eales was born in Belgium in 1993. He received the MPhys degree in physics from the University of Surrey, Surrey, U.K., in 2015. Since 2015, he has been working toward the Ph.D. degree in physics within the photonics group of the Advanced Technology Institute (ATI), University of Surrey. Surrey, U.K. His research has focused on the characterization of mid-infrared III$\mathrm{V}$ semiconductor lasers and silicon compatible photonics.

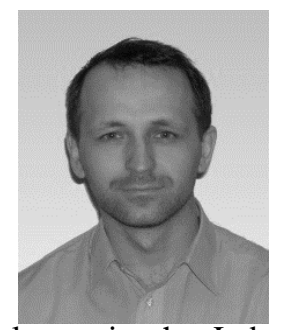

Igor P. Marko was born in Belarus in 1974. He graduated with distinction from the department of quantum radio-physics and optoelectronics of the Belarusian State University in 1996. From 1996 until 2001 he studied the optical properties of $\mathrm{ZnSe}$ and GaN-based wide bandgap semiconductors and optically pumped lasers in the Laboratory of Semiconductor Optics of B. I. 
Stepanov Institute of Physics of the Belarusian Academy of Sciences. He received the $\mathrm{PhD}$ degree in 2000. Since 2001 have been studying the properties of III-V based materials and photonic devices and systems from visible to mid-infrared spectral range at the University of Surrey.

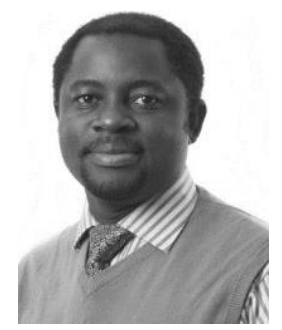

Barnabas Achakpa Ikyo holds a PhD in Physics from the Advanced Technology Institute, University of Surrey Guildford. His interest is in photonics for electrical power generation, environmental and health physics. He has conducted research in measurement characterization and optimization of photonics based devices and their applications in quantum electronics, medical applications and environmental sensing. He has more than 10 year's academic and research experience in physics at University institutions. Currently, he is a Senior Lecturer with the Benue State University Makurdi, Nigeria.

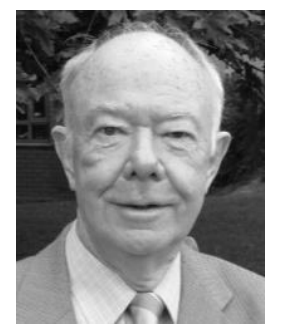

Alfred R. Adams (M '82 SM '00, F'01, LF'06) was born in England in 1939. He received the following degrees in physics from the University of Leicester, UK: BSc, 1961; PhD, 1964; DSc, 1986. After completing his $\mathrm{PhD}$ research into the electrical and optical properties of orthorhombic sulphur he spent two years at the University of Karlsruhe, Germany studying the thermal and thermoelectric properties of selenium and tellurium. In 1967 he joined the University of Surrey where he is now Distinguished Professor of Physics, Emeritus. Semiconductor lasers have been one of his major interests since 1980 when he spent sabbatical leave at Tokyo Institute of Technology and in 1995 he was awarded the Duddell Medal and Prize by the Institute of Physics, UK for proposing inter-valence band absorption and for his work on strained-layers in lasers. He held the Hitachi Visiting Chair at Tokyo Institute of Technology in 1992 and was CNRS Visiting Researcher at the University of Montpellier in 1993. He is a Fellow of the Institute of Physics and an Honorary Fellow of the Institute of Engineering and Technology in the UK. In 1996 he was Elected Fellow of the Royal Society and he was awarded the Rank Prize for optoelectronics in 2014.

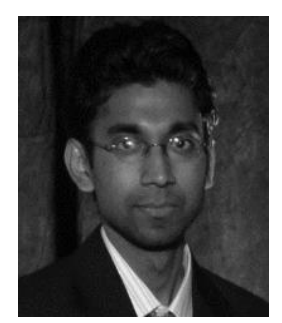

Shamsul Arafin is currently working as an Assistant Project Scientist in the University of California Santa Barbara, CA, USA. Prior to joining UCSB, he worked as a Postdoctoral Research Scholar in Device research laboratory at the University of California Los Angeles, USA. Till now he has authored and coauthored more than 60 papers in leading technical journals and international conferences. He received the IEEE Photonics Society Best Student Paper Award in 2010, IEEE Photonics Society Graduate Student Fellowship in 2011 and Literaturpreis der ITG 2014 Award in 2014. He has also been nominated for the UCLA Chancellor's Award for Postdoctoral Research in 2014. He is a member of the IEEE, IEEE Photonics Society,
Optical Society of America (OSA) and International Society for Optical Engineering (SPIE), and Die Informationstechnische Gesellschaft im VDE (ITG).

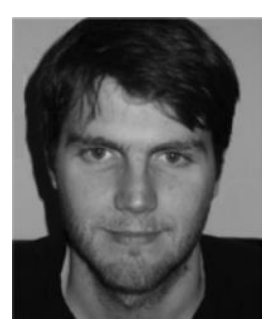

Stephan Sprengel (M'12) was born in Erding, Germany, in 1987. He received the Dipl. Phys. degree in 2012 from the Technische Universität München, Garching, Germany, where he is currently working toward the Ph.D. degree from the Walter Schottky Institut. He is currently involved in the research on type-II InPbased type-I GaSb lasers for the mid-infrared including design, growth, manufacturing, and characterization. Mr. Sprengel is a member of the Deutsche Physikalische Gesellschaft and the IEEE Photonics Society.

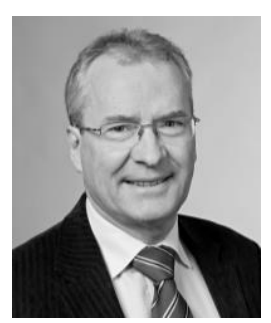

Markus-Christian Amann received the Diplom degree in electrical engineering in 1976 and the Dr.-Ing. degree in 1981, both from the Technische Universität München. From 1981 to 1994 he was with the Corporate Research Laboratories of the Siemens AG in Munich where he was involved in the research on longwavelength InGaAsP-InP laser diodes. In 1994 he joined the Department of Electrical Engineering at the University of Kassel as a full professor for "Technical Electronics". Since 1997 he holds the Chair of "Semiconductor Technology" at the Walter Schottky Institute of the Technische Universität München, where he is currently engaged in the research on tunable laser diodes for the near- and mid- infrared, quantum cascade lasers, long-wavelength vertical-cavity laser-diodes (VCSELs) and laser diode applications. In 2001 he was a cofounder of VERTILAS GmbH, Garching, commercializing long-wavelength VCSELs. He authored or co-authored more than 600 articles (including some 50 invited) on semiconductor optoelectronics in scientific journals and conference proceedings, and co-authored two books. In 1981 he was a recipient of the ITG-prize, in 2004 he won the Karl Heinz Beckurts-prize and in 2009 the Kaiser-Friedrich Research Award. He is a member of the German Informationstechnische Gesellschaft (ITG), and a Fellow of the IEEE Lasers and Electro-Optics Society.

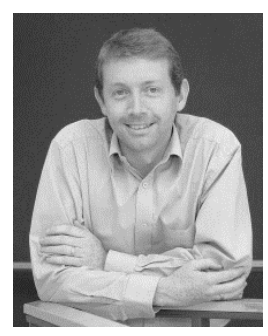

Stephen J. Sweeney (S' 98, M' 99, SM' 06) was born in England in 1973. He received the B.Sc. (Hons.) degree in Applied Physics and the Certificate in Education from the University of Bath, England, in 1995 and the Ph.D. degree in Physics at the University of Surrey, England in 1999. Following post-doctoral research fellow positions working on InGaN LEDs and quantum dot lasers, he became Lead Scientist for Marconi Optical Components working on the development of fixed frequency and tunable lasers for telecommunications applications. He is currently Professor of Physics at the Advanced Technology Institute and Head of the Department of Physics at the University of Surrey. His current research 
interests include novel quantum well and quantum dot materials operating from the visible through to the mid-infrared for applications in solid-state lighting, lasers, detectors and photovoltaics. In addition to his role at Surrey, he is Chief Technology Officer for ZiNIR Ltd., a UK photonics SME, Associate Editor of IEEE Journal of Quantum Electronics and on the Editorial Board of Journal of Materials Science: Materials in Electronics. He is a Chartered Physicist and a Fellow of The Institute of Physics (London). 